\title{
Frequency and voltage control of hybrid AC/DC networks
}

\author{
Jeremy D. Watson and Ioannis Lestas
}

\begin{abstract}
Hybrid AC/DC networks are a key technology for future electrical power systems, due to the increasing number of converter-based loads and distributed energy resources. In this paper, we consider the design of control schemes for hybrid AC/DC networks, focusing especially on the control of the interlinking converters (ILC(s)). We present two control schemes: firstly a decentralized algorithm for primary control, and secondly, a distributed controller for secondary control. In the primary case, the stability of the controlled system is proven in a general hybrid AC/DC network which may include asynchronous AC subsystems. It is shown that power-sharing across the AC/DC network is significantly improved compared to previously proposed dual droop control. The distributed secondary control scheme guarantees the convergence of the $\mathrm{AC}$ system frequencies and the average $\mathrm{DC}$ voltage of each DC subsystem to their nominal values respectively. A prescribed power-sharing is also achieved. The proposed algorithms are verified by simulation on a test hybrid AC/DC network in MATLAB / Simulink.
\end{abstract}

\section{INTRODUCTION}

Hybrid AC/DC networks are a key technology for future electrical power systems. One major reason is the increasing number of converter-interfaced energy sources and loads. Direct current grids have several advantages [1] over traditional AC systems: lower power losses, largely due to the absence of reactive power; higher power transfer capability; and DC grids can also facilitate the connection of asynchronous $\mathrm{AC}$ grids. However, AC technology is well established and is more suitable for some applications. Therefore, it is likely that $\mathrm{AC}$ and $\mathrm{DC}$ technology will be combined via interlinking voltage source converters (VSC) to form a hybrid AC/DC network [2].

Hybrid AC/DC networks present new challenges in terms of frequency and voltage control [3]. In particular, the control of the interlinking converter (ILC) is an important challenge [4] as its operation affects both the AC frequency and the DC voltage. In this paper, we present new voltage source converter control schemes for the interlinking converters which are inspired by the controller proposed in [5]. The proposed controller uses the energy stored in the DC capacitance as the "inertia" for the emulation of synchronous machines. We show that this idea can successfully be applied to the interlinking converter in general hybrid AC/DC networks for both primary and secondary control, and we show that the proposed scheme has advantages over previously proposed ILC controllers.

The paper is organized as follows. Section II presents an overview of control schemes proposed for hybrid AC/DC

J.D. Watson and Ioannis Lestas are with the Department of Engineering, University of Cambridge, Trumpington Street, Cambridge, CB2 1PZ, United Kingdom. This work was partly supported by ERC starting grant 679774. networks, with special attention to the control of the interlinking converter. Section III presents the network model and formulates the control problem. Our main results are given in section IV, including the proposed primary controller in section IV-A and the distributed secondary controller in section IV-B. The performance of the two controllers is illustrated via a case study in section V. Finally, conclusions are presented in section VI.

\section{RELATED WORK}

Decentralized primary control strategies have significant advantages over centralized approaches, including: simplicity, plug-and-play capability, and immunity to communication failure [4]. The most common example, droop control, is cheap, intuitive, and easy to implement. AC frequency droop is ubiquitous, while DC voltage droop controllers are prevalent in the literature as well as in the few industrial multiterminal DC (MTDC) projects [8]. The DC bus voltage dynamics are comparable to traditional AC frequency / real power control, in that an excess of power results in the MTDC voltages rising, while a shortage causes the MTDC voltages to decrease.

Therefore, an obvious way to control the interlinking converter is a dual droop scheme combining the two characteristics in one controller. The DC voltage droop stabilizes the DC grid and the MTDC systems participate in the frequency regulation of the connected $\mathrm{AC}$ grids [9]. However, the two droops interact with each other in a way that degrades their performance, as analyzed by [10], where a modified droop coefficient was calculated to prioritize the AC-side performance. Another approach is seen in [11] which uses receding horizon control to provide a compromise between $\mathrm{AC}$ frequency and DC voltage deviations.

A new idea for the control of the ILCs was proposed in [2], [12], and [13]. The per-unit values of the AC frequency and DC voltage are synchronized by controlling the power transfers with a proportional-integral controller. This allows the ILC to perform network emulation by relating the AC frequencies and DC voltages to each other; and power sharing is then set by the droop coefficients on both the $\mathrm{AC}$ and DC sides. Our proposed method for primary control has a similar aim; however the proposed controller allows faster regulation via the energy stored in DC-side capacitance compared to schemes that directly control the power transfer.

Secondary controllers with communication aim to restore the frequencies and voltages to their nominal value and to share power equitably between sources in the $\mathrm{AC}$ and DC subsystems [3]. In [14], a distributed controller for sharing frequency reserves of asynchronous AC systems via 
HVDC was designed. However the DC voltage dynamics were not modelled. The authors in [15] designed distributed controllers for distributed frequency control of asynchronous AC systems connected through a MTDC grid.

In this paper we will show that the use of a novel VSC controller for the interlinking converter allows general AC/DC networks to be stabilized and optimized and improves the performance in the primary time-frame compared to previous schemes. The main contributions of this paper are as follows:

1) We formulate the primary control problem for the hybrid AC/DC network and propose a controller that is shown to be applicable to this problem. The stability of a general hybrid AC/DC network in the presence of droop-controlled sources is guaranteed while achieving close to optimal power-sharing.

2) We design a distributed secondary controller for both the interlinking converters and the sources which guarantees the convergence of both the AC system frequencies and the weighted average DC voltage of each DC subsystem to their nominal values. It furthermore achieves a prescribed power sharing. We also show that virtual capacitance in the controller can be used to adjust the weights and/or improve performance.

\section{PROBLEM FORMULATION}

\section{A. Network model}

We consider a general hybrid AC/DC network with the set of buses denoted by $N=(1,2, \ldots,|N|)$ and the set of transmission lines by $E=(1,2, \ldots,|E|)$. The network is composed of multiple AC and DC subsystems. We denote the set of subsystems by $K=(1,2, \ldots,|K|)$ and we also have $N=\left(\cup_{i} N_{i}^{d c}\right) \cup\left(\cup_{j} N_{j}^{a c}\right)$, where $N_{i}^{a c}$ and $N_{j}^{d c}$ denote the collection of buses belonging to the AC subsystem $i \in K$ and DC subsystem $j \in K$ respectively. Each subsystem is assumed to be connected and it is connected to the rest of the network only via an interlinking converter ${ }^{1}$, as illustrated in Figure 1. Each AC subsystem $i$ is described by the connected graph $\left(N_{i}^{a c}, E_{i}^{a c}\right)$ with arbitrary direction, and each DC subsystem $j$ by the connected graph $\left(N_{j}^{d c}, E_{j}^{d c}\right)$ with arbitrary direction ${ }^{2}$. For each bus $j \in N$ we use $j: i \rightarrow$ $j$ and $j: j \rightarrow k$ to denote the predecessors and successors of bus $j$ respectively. For convenience we also define the set of all AC buses $N_{a c}=\cup_{i} N_{i}^{a c}$ and all DC buses $N_{d c}=\cup_{j} N_{j}^{a c}$, such that $N_{a c} \cup N_{d c}=N$; likewise we define the set of all AC edges $E_{a c}=\cup_{i} E_{i}^{a c}$ and all DC edges $E_{d c}=\cup_{j} E_{j}^{d c}$, such that $E_{a c} \cup E_{d c}=E$. Connections between AC and DC buses are facilitated by the interlinking converters, the set of which is denoted by $X=(1,2, \ldots,|X|)$. The ILC buses are denoted by $X_{x}^{a c} \in N_{a c}$ and $X_{x}^{d c} \in N_{d c}$ for the AC and DC buses, respectively, to which the ILC $x$ is connected. The set of AC buses to which a converter is connected is denoted by $X_{a c}=\left(X_{1}^{a c}, \ldots, X_{|X|}^{a c}\right) \subset N_{a c}$. Similarly, the

\footnotetext{
${ }^{1}$ Note that this is without loss of generality since the connection of two collections of AC buses (or DC buses respectively) may simply be considered as one larger subsystem.

${ }^{2}$ The results presented in the paper are unaffected by the choice of direction.
}

set of DC buses to which a converter is connected is denoted by $X_{d c}=\left(X_{1}^{d c}, \ldots, X_{|X|}^{d c}\right) \subset N_{d c}$.

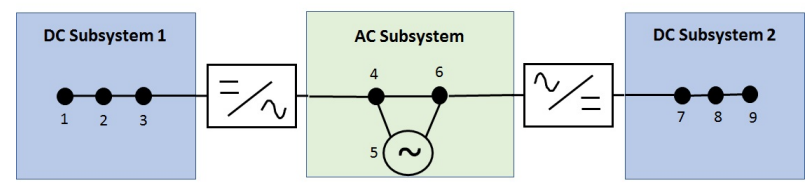

Fig. 1. AC/DC network diagram.

TABLE I

NOTATION IN SYSTEM MODEL

\begin{tabular}{cl}
\hline$\omega_{j}$ & AC frequency deviation at bus $j$ \\
$\eta_{i j}$ & voltage angle between two AC buses $i$ and $j$ \\
$M_{j}$ & inertia at bus $j$ \\
$p_{j}^{G}$ & generated power at bus $j$ \\
$p_{j}^{L}$ & load power at bus $j$ \\
$p_{j}^{X}$ & interlinking converter power transfer at bus $j$ \\
$p_{i j}$ & power transfer between buses $i$ and $j$ \\
$B_{i j}$ & transmission line susceptance for $(i, j) \in E_{a c}$ \\
$D_{j}$ & damping coefficient at bus $j \in N_{a c} \backslash X_{a c}$ \\
$C_{j}$ & capacitance at bus $j \in N_{d c}$ \\
$V_{j}$ & DC voltage deviation at bus $j$ \\
$G_{i j}$ & line conductance for $(i, j) \in E_{d c}$ \\
\hline
\end{tabular}

Assumption 1. We make the following assumptions regarding the network:

- la: Bus voltages have a magnitude of 1 p.u. for all $j \in N_{a c}$.

- 1b: Lines $(i, j) \in E_{a c}$ are lossless and are characterized by their constant reactances $X_{i j}>0$.

- 1c: Reactive power may be ignored as it does not have a significant effect on either bus voltage angles or the frequency.

- 1d: The AC system(s) are three-phase balanced.

- 1e: Bus voltages are close to 1 p.u. for all $j \in N_{d c}$, such that currents and powers are approximately equivalent in a per-unit system.

- If: Lines $(i, j) \in E_{d c}$ are characterized by their conductance $G_{i j}=\frac{1}{R_{i j}}$, where $R_{i j}$ is the line resistance. We also assume that line losses are small and may be neglected.

Remark 1: Assumption 1 may be explained as follows:

- 1a, 1b, 1c, 1d: These are well-known assumptions for AC transmission systems which are used in much of the literature, e.g. [16]. These assumptions allow us to model the active power transfer through a line $(i, j)$ as $p_{i j}=B_{i j} \sin \eta_{i j}$ where $B_{i j}=3 X_{i j}^{-1}>0$.

- 1e, 1f: These are also typical assumptions for DC systems [15].

We also consider the modelling of the interlinking converters, as illustrated in Fig. 2. The AC-side bus of the interlinking converter has no inertia of its own, however power 
imbalances in the AC subsystem lead to a power transfer through the converter. Note also that this power transfer will affect the DC-side voltage of the converter. For each bus $j \in X_{a c} \cup X_{d c}$, we define the power transfer $p_{j}^{X}$ as the power leaving the bus through the ILC. Hence for a converter bus $j \in X_{a c}$, the power transfer $p_{j}^{X}$ is the AC-to-DC transfer, whereas for a converter bus $j \in X_{d c}$, the power transfer $p_{j}^{X}$ is the DC-to-AC transfer. We assume that such power transfers are instantaneous and lossless, hence for an ILC $x$ we have $p_{X_{x}^{d c}}^{X}=-p_{X_{x}^{a c}}^{X}$. For simplicity, we assume that

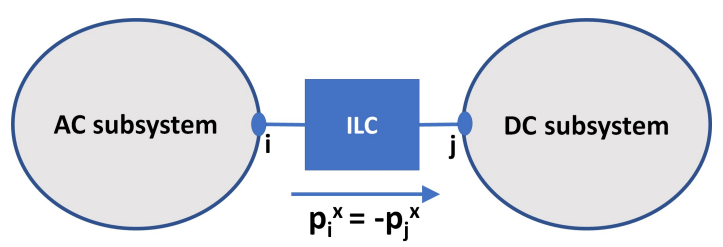

Fig. 2. ILC connection diagram

any generation directly connected to the AC-side bus of the ILC is frequency-independent. Given these assumptions and definitions, the hybrid AC/DC network dynamics are:

$$
\begin{aligned}
& \dot{\eta}_{i j}=\omega_{i}-\omega_{j},(i, j) \in E_{a c} \\
& M_{j} \dot{\omega}_{j}=p_{j}^{G}-p_{j}^{L}+\sum_{j: i \rightarrow j} p_{i j}-\sum_{j: j \rightarrow k} p_{j k}-D_{j} \omega_{j} \text {, } \\
& j \in N_{a c} \backslash X_{a c} \\
& 0=p_{j}^{G}-p_{j}^{L}+\sum_{j: i \rightarrow j} p_{i j}-\sum_{j: j \rightarrow k} p_{j k}-p_{j}^{X}, \\
& j \in X_{a c} \\
& C_{j} \dot{V}_{j}=p_{j}^{G}-p_{j}^{L}+\sum_{j: i \rightarrow j} p_{i j}-\sum_{j: j \rightarrow k} p_{j k}-p_{j}^{X}, \\
& j \in N_{d c} \\
& p_{i j}=\left\{\begin{array}{ll}
B_{i j} \sin \eta_{i j} & j \in N_{a c} \\
G_{i j}\left(V_{i}-V_{j}\right) & j \in N_{d c}
\end{array},(i, j) \in E\right.
\end{aligned}
$$

We now write the system dynamics in matrix form. The vector of angle differences is $\eta=\left[\eta_{i j}\right]_{(i, j) \in E_{a c}}$, the vector of $\mathrm{AC}$ frequency deviations from its nominal value (50 or $60 \mathrm{~Hz})$ is denoted by $\omega=\left[\omega_{j}\right]_{j \in N_{a c}}$, while the vector of DC voltage deviations from their nominal value is denoted by $V=\left[V_{j}\right]_{j \in N_{d c}}$. $M$ is the diagonal matrix of the generator inertias $M_{j}, j \in N_{a c} \backslash X_{a c}$, while the damping coefficients are $D=\operatorname{diag}\left(\left[D_{j}\right]_{j \in N_{a c} \backslash X_{a c}}\right.$. The frequencies at the corresponding AC buses are denoted by $\omega^{G}=\left[\omega_{j}\right]_{j} \in$ $N_{a c} \backslash X_{a c}$, and the vector of frequencies at the converter buses is $\omega^{X}=\left[\omega_{j}\right]_{j \in X_{a c}}$. The diagonal matrix of the DC bus capacitances is $C=\operatorname{diag}\left(\left[C_{j}\right]_{j \in N_{d c}}\right)$. The vector of generator powers is denoted by $p^{G}=\left[p_{j}^{G}\right]_{j \in N}$, the vector of load powers by $p^{L}=\left[p_{j}^{L}\right]_{j \in N}$. We also use the notation $p_{j}^{X}=0$ for buses without converters, i.e. $j \in N \backslash\left(X_{a c} \cup X_{d c}\right)$ and denote the vector of converter powers by $p^{X}=\left[p_{j}^{X}\right]_{j \in N}$. The power transfer vector is defined by $p^{F}=\left[p_{j}^{F}\right]_{j \in N}$, where each $p_{j}^{F}=\sum_{j: i \rightarrow j} p_{i j}-\sum_{j: j \rightarrow k} p_{j k}$. The matrix $A$ is the incidence matrix of the graph $\left(N_{a c}, E_{a c}\right)$. The system equations then become:

$$
\begin{gathered}
\dot{\eta}=A^{T} \omega=A^{T}\left[\begin{array}{c}
\omega^{G} \\
\omega^{X}
\end{array}\right] \\
{\left[\begin{array}{c}
M \dot{\omega}^{G} \\
0 \\
C \dot{V}
\end{array}\right]=p^{G}-p^{L}-p^{X}-p^{F}-\left[\begin{array}{c}
D \omega^{G} \\
0 \\
0
\end{array}\right]}
\end{gathered}
$$

1) Equilibrium conditions: An equilibrium of the system in (2) is defined by the following conditions:

$$
\begin{aligned}
& 0=A^{T} \omega=A^{T}\left[\begin{array}{c}
\omega^{G} \\
\omega^{X}
\end{array}\right] \\
& 0=p^{G}-p^{L}-p^{X}-p^{F}-\left[\begin{array}{c}
D \omega^{G} \\
0 \\
0
\end{array}\right]
\end{aligned}
$$

We assume that there exists some equilibrium point of (2), and denote such an equilibrium by $\left(\eta^{*}, \omega^{G *}, V^{*}\right)$. Individual equilibrium values are also denoted by the superscript asterisk, e.g. $\eta_{i j}^{*}, \omega_{i}^{G *}$, and $V_{j}^{*}$.

Assumption 2. $\left|\eta_{i j}^{*}\right|<\frac{\pi}{2}$ for all $(i, j) \in E_{a c}$.

This assumption is often referred as a security constraint and is common in the literature for power grid stability analysis.

\section{B. Control objectives}

The control objectives are:

1) Solutions must converge to an equilibrium point.

2) For primary control, AC frequencies and DC voltages should not deviate significantly from their nominal values at steady-state.

3) $\mathrm{AC}$ frequencies must converge to their nominal values for secondary control.

4) The power sharing between AC/DC sources should be optimal.

The last objective may be stated more formally by considering the minimization of a quadratic cost function [6]:

$$
\begin{gathered}
\min _{p^{G}} C_{G}=\frac{1}{2}\left(p^{G}\right)^{T} Q p^{G} \\
\text { subject to: } \mathbf{1}^{T} p^{G}=\mathbf{1}^{T} p^{L}+\mathbf{1}^{T}\left[\begin{array}{c}
D \omega^{G} \\
0 \\
0
\end{array}\right]
\end{gathered}
$$

where $Q$ is a diagonal matrix containing the cost coefficients for each energy resource, and $\mathbf{1}$ is the vector of ones. For convenience we also write the decomposition of $Q$ into its corresponding $\mathrm{AC}$ and $\mathrm{DC}$ sources as $Q=\operatorname{diag}\left(Q_{a c}, Q_{d c}\right)$ where $Q_{a c}$ and $Q_{d c}$ are diagonal matrices containing the cost coefficients for the AC and DC generators respectively. We define the corresponding entries in the inverse matrix $\left[Q^{-1}\right]_{i i}$ as the reciprocal of $Q_{i i}$ if $Q_{i i} \neq 0$, and zero otherwise. Using the standard method of Lagrange multipliers as in [6], and defining the vector 
$p^{u}=\left[\begin{array}{lll}D\left(\omega^{G}\right)^{T} & 0^{T} & 0^{T}\end{array}\right]^{T}$ for convenience, the solution $p^{G *}$ to the optimization problem is:

$$
p^{G *}=\frac{Q^{-1} \mathbf{1 1}^{T}}{\mathbf{1}^{T} Q^{-1} \mathbf{1}}\left(p^{L}+p^{u}\right)
$$

\section{MAIN RESUlTS}

In this section we present our main results. The proofs of the stated theorems have been omitted due to space constraints but may be found in the extended version of this paper.

\section{A. Decentralized primary control}

We assume the use of power-frequency droop control for the AC generators and power-voltage droop for the DC energy resources, i.e.:

$$
p^{G}=-Q^{-1}\left[\begin{array}{c}
\omega \\
m V
\end{array}\right]+p_{\text {nom }}^{G}
$$

where $Q^{-1} \geq 0$ is the inverse cost matrix of droop coefficients, and $m>0$ is a constant, and as stated previously, $\omega$ and $V$ are the column vectors of the AC frequency and DC voltage deviations, respectively. The nominal power generation $p_{n o m}^{G}$ is the power allocation that satisfies (4) when the frequencies are at their nominal values. The second control objective (limitation of frequencies and voltages deviations) may be satisfied by choosing suitably large droop coefficients in $Q^{-1}$.

We also introduce a VSC controller which is based on [5]. Let the voltage angle $\theta_{i}$ at the AC-side output of an ILC $x$ be given by

$$
\begin{aligned}
\theta_{i} & =\int m V_{j}, \text { i.e. the frequency is given by, } \\
\omega_{i} & =\dot{\theta}_{i}=m V_{j}
\end{aligned}
$$

where $i \in X_{x}^{a c}$ and $j \in X_{x}^{d c}$. This relates the AC frequency deviation proportionally to the DC voltage deviation by a chosen constant $m>0$. We assume that the converter is lossless and that the internal dynamics are sufficiently fast compared to the network dynamics. In [5] the suggestion is to set $m=\frac{\omega^{n o m}}{V_{d d}^{n o m}}$ where $\omega^{\text {nom }}$ and $V_{d c}^{n o m}$ are the nominal values of the $\AA C$ grid frequency and the DC grid voltage. Since in this paper $\omega_{i}$ and $V_{j}$ are deviations from a nominal value, other values of $m$ are also possible. Large values of $m$ result in smaller DC voltage deviations and larger AC frequency deviations, and in general $m=\frac{\omega^{n o m}}{V_{d c}^{n o m}}$ may be too large for this scheme as frequency deviations are generally less acceptable than voltage deviations.

Instead of directly controlling the power transfer, (7b) relates the frequency and voltage within the $\mathrm{AC}$ and $\mathrm{DC}$ sides, respectively. Not only does this improve the accuracy of the power-sharing in the primary time-frame, but a further advantage is the fast response to $\mathrm{AC}$ disturbances via capacitive inertia as discussed in [5] and [6]. ${ }^{3}$

\footnotetext{
${ }^{3}$ In this paper, we take this concept further and use the capacitive inertia of the entire DC subsystem for frequency support, and also use the inertia of the AC system to regulate the DC voltage when appropriate.
}

Theorem 1 (Stability): Consider a dynamical system described by equations (1), (2) with the control scheme in (6), and (7b), and an equilibrium point for which Assumption 2 holds. Then an open neighbourhood $S$ exists about this point such that all solutions of the states $\left(\eta, \omega^{G}, V\right)$ with initial conditions in $S$ converge to the set of equilibrium points as defined in (3).

Theorem 2 (Power sharing): As the line resistances become arbitrarily small, the power sharing of the control scheme (6), (7b) becomes arbitrarily close to the solution of the optimization problem (4).

Remark 2: In a practical network there will always be some small line resistances which affect power sharing [17]. Nevertheless, if these resistances are small, voltage disturbances will also be small and the power sharing will be close to optimal.

Remark 3 (Power sharing in a dual-droop ILC controller scheme): Consider the dual-droop scheme (8) sometimes used in the literature for primary control of the ILC,

$$
p_{i}^{X}=K_{i}^{\omega} \omega_{i}-K_{j}^{V} V_{j}
$$

where $K_{i}^{\omega}$ and $K_{j}^{V}$ are the respective droop coefficients, and the power transfer is directly controlled ${ }^{4}$. It is clear that (8) is unable to guarantee correct power-sharing for a disturbance at any arbitrary bus under the same assumptions. For droop-controlled sources to contribute power in proportion to their droop coefficients, a system-wide synchronizing variable is required. The proposed controller (7b) achieves this by relating the AC frequency to the DC voltages. By contrast, the dual droop controller (8) does not provide such a relation.

\section{B. Distributed secondary control}

In this section we propose a distributed controller inspired by [6] and [7]. The concept of network emulation can be carried further with the aid of distributed communication. Let the average DC voltage deviation of each subsystem $k$ be represented by the capacitance-weighted average $\bar{V}_{k}$ :

$$
\bar{V}_{k}=\sum_{j \in N_{k}^{d c}} C_{j} V_{j}
$$

As in the second distributed MTDC controller proposed in [7], the DC voltages within each subsystem are either communicated within the network so as to obtain $\bar{V}_{k}$ (for small subsystems) or $\bar{V}_{k}$ is obtained via an appropriately fast distributed approach, such that its dynamics can be decoupled from the stability analysis in this paper. From (9) we have:

$$
\begin{aligned}
\dot{\bar{V}}_{k} & =\sum_{j \in N_{k}^{d c}} C_{j} \dot{V}_{j} \\
& =\sum_{j \in N_{k}^{d c}}\left(p_{j}^{G}-p_{j}^{L}-p_{j}^{X}+p_{j}^{F}\right)
\end{aligned}
$$

\footnotetext{
${ }^{4}$ In practice, the ILC controls the power transfer by varying its output voltage angle until (8) is satisfied.
} 
The DC branch-flows $p_{j}^{F}$ cancel out within the subsystem, and we therefore have the following expression which resembles the swing equation:

$$
\dot{\bar{V}}_{k}=\sum_{j \in N_{k}^{d c}}\left(p_{j}^{G}-p_{j}^{L}-p_{j}^{X}\right)
$$

We now introduce the concept of virtual frequency deviation $\hat{\omega}$ which is defined for the entire network as follows:

$$
\hat{\omega}_{j}= \begin{cases}\omega_{j} & \text { if } j \in N_{a c} \\ m \bar{V}_{k} & \text { if } j \in N_{k}^{d c}\end{cases}
$$

where $k$ is the DC subsystem to which all nodes in the associated set $N_{k}^{d c}$ belong. We will denote the vector of average DC subsystem voltages by $\bar{V}$. The converter which interlinks AC bus $i$ and the DC subsystem $k$ is governed by

$$
\omega_{i}=m \bar{V}_{k}
$$

where $m>0$ is a positive coefficient. A common approach to achieve power sharing in secondary control is to introduce a synchronizing communicating variable $\xi$, e.g. [6], and update these values via distributed averaging through an undirected connected communication graph with Laplacian $\mathcal{L}$. Then the distributed controllers for the hybrid AC/DC system are:

$$
\begin{aligned}
\tau_{\xi} \dot{\xi} & =-\mathcal{L} \xi-\left[\begin{array}{cc}
I & 0 \\
0 & H
\end{array}\right]^{T} Q^{-1}(\hat{\omega}) \\
p^{G} & =Q^{-1}\left[\begin{array}{cc}
I & 0 \\
0 & H
\end{array}\right] \xi
\end{aligned}
$$

where $\tau_{\xi}$ is a time constant, $\xi$ is the column vector of the synchronizing variables $\xi_{j}, I$ is the square identity matrix with dimension $\left|N_{a c}\right|$ and $Q^{-1}$ is the inverse cost coefficient matrix as before. $H$ is the $\left|N_{d c}\right|$ by $|K|$ connection matrix which maps the DC nodes to the DC subsystems as follows:

$$
H_{j k}=\left\{\begin{array}{lc}
1 & \text { if } j \in N_{k}^{d c} \\
0 & \text { otherwise }
\end{array}\right.
$$

Note that it is now sufficient to consider the system with states $\left(\eta, \omega^{G}, \bar{V}, \xi\right)$, since the local DC voltages are replaced by $\bar{V}$ in the corresponding control mechanisms in (12), (13), (14), in conjunction with the fact that the $\bar{V}$ dynamics in (10) have no explicit dependence on $V$. The DC bus voltages are weighted by the associated capacitances in order to capture the dynamics of the physical energy of the subsystem as follows from (10). An objection might be that buses with low capacitance could have voltages far from the nominal while still satisfying $\bar{V}_{k}=0$. However, since the DC bus voltages must still satisfy the power-flow equations, the voltages of buses with low capacitance will still be close to nominal. Furthermore, virtual capacitance $C_{j}^{V}$ may easily be added at any DC source bus $j$ via a derivative term in the DC source dynamics, e.g.:

$$
p_{j}^{G}=\left[Q^{-1}\right]_{j j} \xi_{j}-C_{j}^{V} \dot{V}_{j}
$$

Theorem 3 (Stability): Consider the dynamical system described in (1),(2) with the control scheme (12), (13), (14) and an equilibrium point for which Assumptions 2 is satisfied. An open neighbourhood $S$ exists about this point such that all trajectories of the states $\left(\eta, \omega^{G}, \bar{V}, \xi\right)$ with initial conditions in $S$ converge to the set of equilibrium points, which satisfy $\omega^{*}=0$ and $\bar{V}^{*}=0$.

Theorem 4 (Power sharing): An equilibrium of the system (2) with the control scheme (12), (13), (14) solves the optimization problem (4).

\section{CAse studies}

In order to demonstrate our results, we study a simple hybrid AC/DC system in MATLAB / Simulink. The parameters of the network are given in Table II. We choose, $m=0.1$, (7b) for the ILCs and thus set the droop controllers in $Q^{-1}$ to have equivalent gains $(40 k W / \omega, 4 k W / V)$. Hence the desired power sharing is equal contributions from all sources. The topology of the test network is shown in Fig. 1.

TABLE II

HYBRID AC/DC NETWORK PARAMETERS

\begin{tabular}{c|c|c} 
Description & Parameter & Value \\
\hline Bus capacitance & $C_{1}, C_{3}, C_{7}, C_{9}$ & $5 \mathrm{mF}$ \\
DC load resistances & $R_{1}, R_{3}, R_{7}, R_{9}$ & $40 \Omega$ \\
DC load resistances & $R_{2}, R_{8}$ & $120 \Omega$ \\
DC line resistances & $R_{12}, R_{89}$ & $0.002 \Omega$ \\
DC line resistances & $R_{23}, R_{78}$ & $0.01 \Omega, 0.02 \Omega$ \\
Rated DC voltage & $V_{d c}^{*}$ & $400 \mathrm{~V}$ \\
AC line constants & $B_{i j}$ & $1 \mathrm{~S}$ \\
Rated AC frequency & $f=\frac{\omega}{2 \pi}$ & $60 \mathrm{~Hz}$
\end{tabular}

\section{A. Decentralized primary control}

Using the controllers (6), (7b) we show that the voltages and frequencies converge to equilibrium values and that the power-sharing is close to the optimal values irrespective of the location of the disturbance. In Fig. 3 we show the AC frequency and DC voltage response to step disturbances at time $t=11 \mathrm{~s}$ and $t=21 \mathrm{~s}$. The magnitude of the earlier disturbance is $4 \mathrm{~kW}$ (nominal added demand) located at bus 9 within DC subsystem 2, while the latter disturbance is 4 $\mathrm{kW}$ reduced demand at bus 1 within DC subsystem 1 . The voltages and frequencies of both the ILC buses converge to identical values as expected. Fig. 4 shows that the required power is shared reasonably accurately. The error is due to the line resistances of the MTDC network.

\section{B. Distributed control}

We also consider the performance of the distributed controllers $(13,14)$ under the same conditions. Figs. 5-6 show that the distributed controller successfully regulates the voltages and frequencies of the hybrid AC/DC network to their nominal values while guaranteeing exact optimal power-sharing at steady-state.

\section{CONCLUSION}

We have proposed a new method for the control of interlinking converter(s), used in conjunction with traditional droop control to guarantee stability and accurate power sharing in a general hybrid AC/DC network. The stability of the 


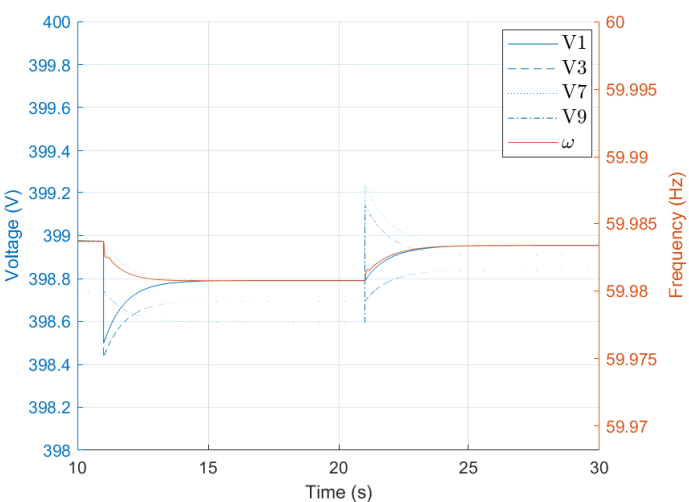

Fig. 3. Frequency and voltage response of the system with decentralized primary control

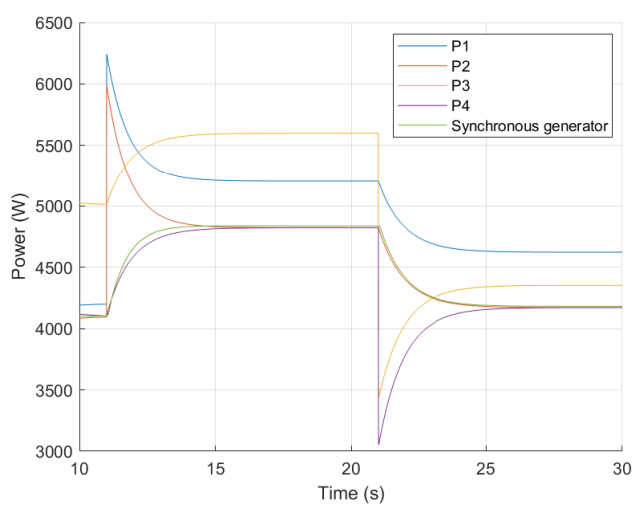

Fig. 4. Power-sharing response of the system with decentralized primary control

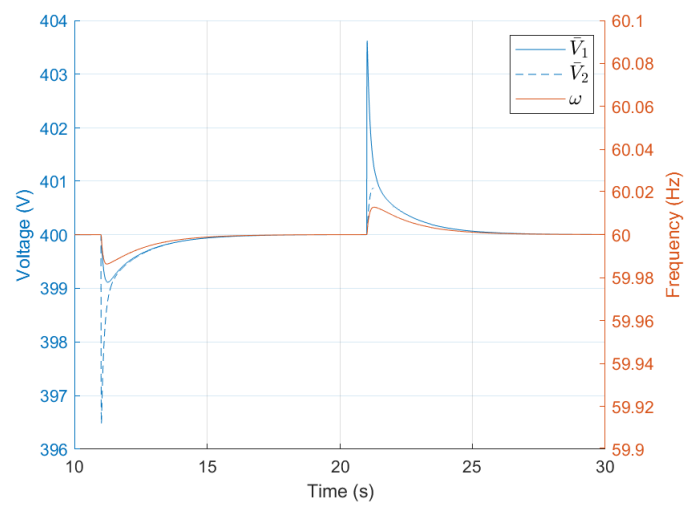

Fig. 5. Frequency and voltage response of the system with distributed control

controlled system was proven and it was shown that powersharing across the AC/DC network is significantly improved compared to dual droop control. A secondary control scheme has also been proposed that guarantees system stability while achieving exact optimal power sharing and the returning of the bus frequencies and the weighted average of DC voltages to their nominal values. Finally, the proposed algorithms were verified by simulation.

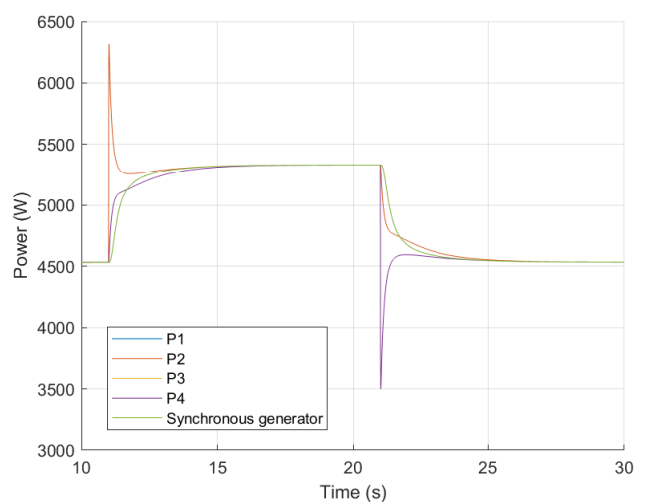

Fig. 6. Power-sharing response of the system with distributed control

\section{REFERENCES}

[1] D. Jovcic et al, "Feasibility of DC Transmission Networks," 2011 IEEE Conference on Innovative Smart Grid Technologies Europe (ISGT Europe), 5-7 Dec. 2011.

[2] P. Wang et al, "Chapter 2 Hybrid AC/DC Micro-Grids: Solution for High Efficiency Future Power Systems," in N.R. Karki et al. (eds.), Sustainable Power Systems, Reliable and Sustainable Electric Power and Energy Systems Management, Springer, 2017.

[3] S.M. Malik et al, "Voltage and frequency control strategies of hybrid AC/DC microgrid: a review," IET Generation, Transmission and Distribution, vol. 11, no. 2, 2017, pp. 303-313.

[4] E. Unamuno, J.A. Barrena, "Hybrid ac/dc microgrids - Part II: Review and classification of control strategies," Renewable and Sustainable Energy Reviews, vol. 52, pp. 1123-1134, December 2015.

[5] T. Jouini et al, "Grid-Friendly Matching of Synchronous Machines by Tapping into the DC Storage," IFAC-PapersOnLine, vol. 49, issue 22, 2016, pp. 192-197.

[6] P. Monshizadeh, et al, "Stability and Frequency Regulation of Inverters with Capacitive Inertia," arXiv:1704.01545v1, 2017.

[7] M. Andreasson et al, "Distributed Controllers for Multi-Terminal HVDC Transmission Systems," IEEE Transactions on Control of Network Systems, vol. 4, no. 3, pp. 564-574, Sept. 2017.

[8] C. E. Spallarossa et al, "A DC Voltage Control Strategy for MMC MTDC Grids incorporating Multiple Master Stations," 2014 IEEE PES T\&D Conference and Exposition, 14-17 April 2014.

[9] N. Chaudhuri, R. Majumder, and B. Chaudhuri, "System frequency support through multi-terminal DC (MTDC) grids," Power Systems, IEEE Transactions on, vol. 28, pp. 347356, Feb 2013.

[10] S. Akkari et al, "Interaction between the Voltage-Droop and the Frequency-Droop Control for Multi-Terminal HVDC Systems," AC and DC Power Transmission, 11th IET International Conference on, 2015.

[11] L. Papangelis et al, "A receding horizon approach to incorporate frequency support into the AC/DC converters of a multi-terminal DC grid," Electric Power Systems Research, vol. 148, 2017, pp. 1-9.

[12] P. C. Loh et al, "Autonomous Control of Interlinking Converter With Energy Storage in Hybrid ACDC Microgrid," in IEEE Transactions on Industry Applications, vol. 49, no. 3, pp. 1374-1382, May-June 2013.

[13] F. Luo et al, "A Hybrid AC/DC Microgrid Control Scheme With Voltage-Source Inverter-Controlled Interlinking Converters", Power Electronics and Applications (EPE'16 ECCE Europe), 18th European Conference on, 2016.

[14] J. Dai and G. Damm, "An improved control law using HVDC systems for frequency control," in Proc. Power Syst. Comput. Conf., 2011.

[15] M. Andreasson et al, "Distributed Frequency Control Through MTDC Transmission Systems," IEEE Transactions on Power Systems, vol. 32, no. 1, Jan. 2017, pp. 250-260.

[16] A. Kasis, E. Devane, C. Spanias, and I. Lestas, "Primary frequency regulation with load-side participation - Part I: stability and optimality," IEEE Trans. Power Systems, vol. 32, no. 5, pp. 3505-3518, Sept. 2017.

[17] T.M. Haileselassie and K. Uhlen, "Impact of DC Line Voltage Drops on Power Flow of MTDC Using Droop Control," IEEE Transactions on Power Systems, vol. 27, no. 2, August 2012, pp. 1441-1449. 